\title{
Metrological characteristics of the torque measurement of electric motors
}

\author{
Oleksandr M. Vasilevskyi* \\ Department of Metrology and Industrial Automation, Vinnytsia National Technical University, 95 Khmelnitskoye Shose, \\ Vinnytsia 21021, Ukraine
}

Received: 21 July 2015 / Accepted: 25 January 2017

\begin{abstract}
This paper proposes a method of determination of the basic metrological characteristics of measurement means (MM) in order to measure the torque of electric motors (EM), such as sensitivity, nominal conversion functions, and additive and multiplicative errors. The nature of change of metrological characteristics was researched using the Measurand Model derived, in the conversion measuring range studied in this paper. Summarised values for additive and multiplicative errors were established for the MM of torque, under the conditions of deviation in terms of the impact value from its nominal value. In addition, the methodology was analysed to enable recalculation of additive and multiplicative errors related to the standard uncertainty of type B. This was undertaken to establish the quality of measurement according to international standards. The research was founded on Measurand Model obtained for multiplicative and additive components in the errors inherent in torque MM.
\end{abstract}

Keywords: torque / sensitivity / additive errors / multiplicative errors / standard uncertainty

\section{Introduction}

This research was conducted into the basic metrological characteristics displayed when developing new designs of measurement means (MM) for the evaluation of physical quantities. This is seen to be a crucial issue in science, the solution of which contributes to ensuring both uniformity of measurements as well as in the identification of primary and secondary uncertainties in various types of measurement [1-6].

As has been demonstrated in the relevant literature [4-14], the basic characteristics of MM include sensitivity, additive errors, multiplicative errors, measurement ranges, and primary and secondary errors (uncertainties) in the MM which in international scientific metrology practice is sometimes known as the Measurand Model. These characteristics are sufficient to establish standards for the accuracy of the MM $[11,12]$. This requires that the mathematical tools used in the research study of a MM in static mode (i.e. steady state) enable us to easily obtain functions of the underlying metrological characteristics as previously enumerated. The difficulty is that the equations describing measurement conversion describe different physical processes for different MMs, which relate to a variety of disciplines and are functions

* Corresponding author: o.vasilevskyi@gmail.com of numerous variables. It would be valuable to produce a generalised method of research, independent of the physical phenomena underlying the design of MMs. This research aims to achieve this, by appraising a series of derived conversion equations using the Taylor's Series method to study static metrological characteristics by mathematical means.

The aim of this paper is to develop a methodology to refine the calculation of analytical expressions for the additive errors and multiplicative errors present in a torque measurement model of electric motors (EM) in terms of an analysis of the model's associated Taylor series in order to estimate the model's a priori type B uncertainties.

\section{Equation for the conversion of the torque measuring means}

A structural diagram of the MM was developed which is shown in Figure 1 to measure the torque of EMs. The components of the means for measuring the torque of motors include a drive EM (DM) that is connected to the test motor (MT) via the coupling conjugation (CC), a measuring transducer for angular speeds in voltage $(\omega / U)$, a torque converter into an angular position (a measuring lever) $\left(\mathrm{M}_{P} / \phi\right)$, a rotation angle converter $(\phi / Q)$, an energy converter in voltage $(Q / U)$, scale converters (SC1, SC2), a 


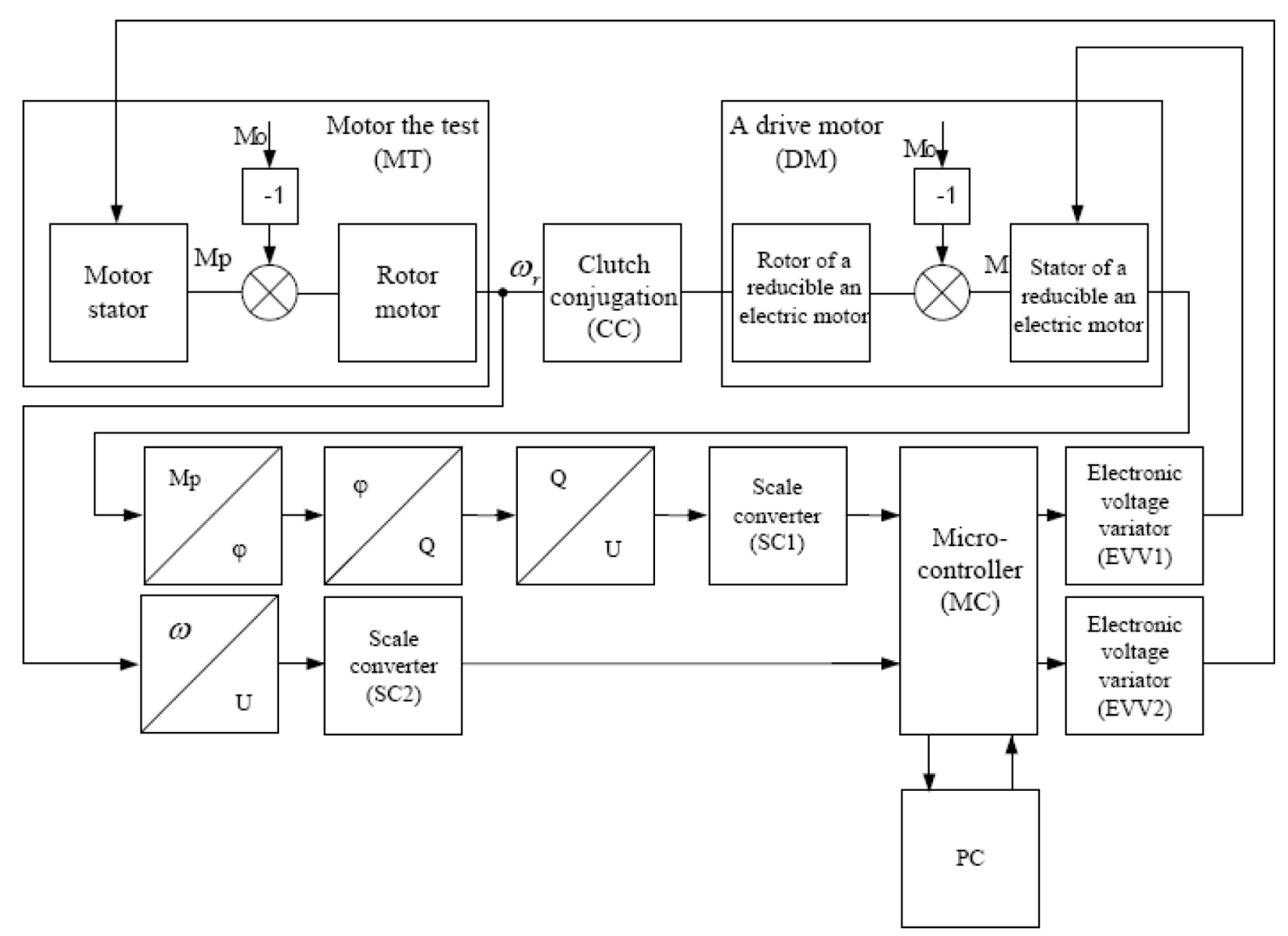

Fig. 1. Block diagram of the torque MM.

microcontroller with built-in analogue-to-digital converter (ADC) (MC), electronic voltage variators (EVV1, EVV2) and a personal computer [7].

The essence of the method of measuring the torque of the test motor is to use a drive motor to generate a slow rotation of the shaft of the test motor with an equivalent frequency corresponding to $0.2-0.3$ of the nominal rotational speed of the test motor. Under these conditions, the test motor is provided with nominal voltage and frequency. This design allows the dependence of the minimum and the maximum torque of the test motor to be obtained, depending on the angular position of the rotor.

Using the analytical expression of the conversion function of a MM of the torque of EMs (Fig. 1) in static mode and the standard equation for conversion by an ADC, we obtain this equation for the conversion of a torque MM, which we may use to obtain basic metrological characteristics

$$
N_{\mathrm{My}}=A\left(K 1 \frac{S+(B / J)\left(M_{P}-M_{\mathrm{CC}}\right)}{(C J-D)}+\left(M_{P}-M_{\mathrm{CC}}\right)\right) \text {, }
$$

where $A=U_{p} 2^{m} / 4 g l R S_{C} U_{\mathrm{o}} ; B=T_{s} p / i \eta \omega_{r} ; \quad C=\gamma \omega^{2}$; $D=\omega^{2} T_{s} p / \omega_{r} ; U_{p}$ is the ADC supply voltage; $U_{o}$ is the $\mathrm{ADC}$ reference voltage; $m$ is the ADC digital capacity; $g$ is the acceleration of gravity; $l$ is the length of the measurement arm; $R$ is resistance value of the tensoresistive bridge; $S_{C}$ is the value of the sensitivity of the effort sensor; $\omega$ is the free natural frequency (undamped) of the fluctuations of the transformer; $K 1$ is the value of the coefficient of the conversion of the tensoresistive transformer; $S$ is the slip; $J$ is the moment of inertia of the moving part of the EM; $p$ is the number of pole pairs of the EM; $i$ is the transmission value of the reductor; $\eta$ is the efficiency coefficient of the reductor; $\omega_{r}$ is the angular speed of the EM rotor; $\mathrm{M}_{\mathrm{CC}}$ is the moment of eccentricity of the clutch coupling; $T_{s}$ is the electromagnetic time constant of the stator; $\gamma$ is the value of the coefficient of the slope of the slip characteristics; $\mathbf{M}_{P}$ is the torque of the EM - measured value (Tab. 1).

\section{The metrological characteristics of the Torque Measurement Means}

Under the working conditions of the MM, the torque provides functional conversion of the informative (useful) parameter $\mathrm{M}_{P}$ in the source code $N_{\mathrm{My}}$. In addition to the informative signal, it is also influenced by impact values which include all the other parameters that have a direct relationship with the original value and causing uninformative (stray) components of the conversion. Analysis of the derived conversion equation (1) demonstrates that the process of the conversion of informative parameter in the output signal is influenced by a large number of parameters (impact values). But due to the fact that almost all the values are unchanged, and one of the most important parameters when testing the EM in addition is the torque is the moment of inertia, we will explore the impact of the moment of inertia as an impact value on the informative parameter. 
Table 1. Defining the terms.

\begin{tabular}{lll}
\hline Designation & Name & Defining \\
\hline$U_{p}$ & ADC supply voltage & $\begin{array}{l}\text { A supply voltage is an electronic device that supplies } \\
\text { electric energy to an electrical load. The primary function } \\
\text { of a power supply is to convert one form of electrical } \\
\text { energy to another and, as a result, power supplies are } \\
\text { sometimes referred to as electric power converters }\end{array}$ \\
& $\begin{array}{l}\text { A voltage reference is an electronic device that ideally } \\
\text { produces a fixed (constant) voltage irrespective of the }\end{array}$ \\
& $\begin{array}{l}\text { loading on the device, power supply variations, } \\
\text { temperature changes, and the passage of time. Voltage } \\
U_{\mathrm{O}}\end{array}$ & $\begin{array}{l}\text { references are used in power supplies, analog-to-digital } \\
\text { converters, digital-to-analog converters, and other } \\
\text { measurement and control systems }\end{array}$
\end{tabular}

The digital capacity of the converter indicates the number of discrete values it can produce over the range of analog values. The resolution determines the magnitude of the quantization error and therefore determines the maximum possible average signal to noise ratio for an ideal ADC without the use of oversampling

The standard acceleration due to gravity (or standard acceleration of free fall), sometimes abbreviated as standard gravity, is the nominal gravitational acceleration of an object in a vacuum near the surface of the Earth $\left(9.80665 \mathrm{~m} / \mathrm{s}^{2}\right)$.

A measurement arm (lever) is a rigid body capable of rotating on a point on itself.

A tensoresistive bridge is an electrical circuit used to measure an unknown electrical resistance by balancing two legs of a bridge circuit

$S_{C} \quad$ Sensitivity of the effort sensor

A sensor's sensitivity indicates how much the sensor's output changes when the input quantity being measured changes moving part of the electric motor

Natural frequency is the frequency at which a system tends to oscillate in the absence of any driving or damping force

A proportionality coefficient of linear dependence of input $\left(M_{p}\right)$ and output $\left(N_{\mathrm{My}}\right)$ signals

Slip is defined as the difference between synchronous speed and operating speed, at the same frequency, expressed in rpm or in percent or ratio of synchronous speed

The mass moment of inertia measures the extent to which an object resists rotational acceleration about a particular axis, and is the rotational analogue to mass

Any node of a circuit that is available for connection to an external circuit is called a pole (or terminal if it is a physical object). The port condition is that a pair of poles of a circuit is considered a port if and only if the current flowing into one pole from outside the circuit is equal to the current flowing out of the other pole into the external circuit 
Table 1. (continued).

\begin{tabular}{lll}
\hline Designation & Name & Defining \\
\hline$i$ & $\begin{array}{l}\text { Transmission value of the } \\
\text { reductor }\end{array}$ & $\begin{array}{l}\text { Transmission ratio is formally considered as the ratio of } \\
\text { the number of the wheel teeth to the number of gear teeth }\end{array}$ \\
& $\begin{array}{l}\text { Efficiency coefficient } \\
\text { system (devices, machines), or in respect of the conversion } \\
\text { of energy transfer. It determines the ratio of useful energy } \\
\text { used to the total amount of energy received by the system } \\
\text { (device, machine) }\end{array}$ \\
& $\begin{array}{l}\text { The angular speed is defined as the rates of change of } \\
\text { angular speed } \\
\omega_{r}\end{array}$ & $\begin{array}{l}\text { precisely, a pseudovector) that specifies the angular speed } \\
\text { (rotational speed) of an object and the axis about which } \\
\text { the object is rotating }\end{array}$ \\
&
\end{tabular}

$\mathrm{M}_{\mathrm{CC}} \quad$ Moment of eccentricity of the clutch coupling

$T_{s} \quad$ Electromagnetic time constant of the stator

$\mathrm{M}_{P} \quad$ Torque of the electric motor

$S_{P} \quad$ Sensitivity of means of measurement

$\Delta N_{J} \quad$ Multiplicative error

$\Delta N_{a J} \quad$ Additive error

$u_{\text {Binst }} \quad$ Uncertainty of type B
A coupling is a device used to connect two shafts together at their ends for the purpose of transmitting power. Couplings do not normally allow disconnection of shafts during operation, however there are torque limiting couplings which can slip or disconnect when some torque limit is exceeded

Eccentricity - numerical characteristic of a conic section, showing the extent of its deviation from the circle

Electromagnetic time constant of the stator is associated to the cutoff frequency of the stator field and the critical slip value

A torque motor is a specialized form of electric motor which can operate indefinitely while stalled, that is, with the rotor blocked from turning, without incurring damage. In this mode of operation, the motor will apply a steady torque to the load (hence the name). A torque motor that cannot perform a complete rotation is known as a limited angle torque motor

The sensitivity of means of measurement - measuring means property defined by the ratio of change of the means of the output signal $\left(N_{\mathrm{My}}\right)$ to the calling of his change of the measured value $\left(\mathbf{M}_{P}\right)$.

Multiplicative called error linearly increasing or decreasing with the increase of the measured value (the error is proportional to the measured value)

Additive error called error constant at each point of the scale

Uncertainty of type B is a component of uncertainty to which the statistical evaluation methods cannot be applied. In such cases, use other known methods (scientific judgments, which are based on accessible information about the possible changes of the measured value) 
In order to evaluate the accuracy of the MM in the static mode of operation, we should use a universal mathematical tool that permits us to simply form basic models of the metrological characteristics of the MM. We used the mathematical tool of transformation equations) in Taylor's Series, using the decomposition of conversion equation. As a result of the decomposition of the transformation equation (1) in a Taylor's 2 Series, we obtain

$$
\begin{aligned}
N_{\mathrm{My}} & =N_{\mathrm{My} 0}+\left[\frac{\partial N_{\mathrm{My}}}{\partial M_{P}}\right]_{0} M_{P}+\frac{1}{2 !}\left[\frac{\partial^{2} N_{\mathrm{My}}}{\partial M_{P}^{2}}\right]_{0} M_{P}^{2} \\
& +\frac{1}{3 !}\left[\frac{\partial^{3} N_{\mathrm{My}}}{\partial M_{P}{ }^{3}}\right]_{0} M_{P}^{3}+\cdots+\left[\frac{\partial^{2} N_{\mathrm{My}}}{\partial M_{P} \partial J}\right]_{0} M_{P} \Delta J \\
& +\left[\frac{\partial N_{\mathrm{My}}}{\partial J}\right]_{0} \Delta J+\frac{1}{2 !}\left[\frac{\partial^{2} N_{\mathrm{My}}}{\partial J^{2}}\right]_{0} \Delta J^{2}+\cdots .
\end{aligned}
$$

The analytical expression of the sensitivity of the torque MM has the form

$$
S_{M_{P}}=\frac{\partial N_{\mathrm{My}}}{\partial M_{P}}=A\left(\frac{B \times K 1}{J(J C-D)}+1\right) .
$$

Changes in sensitivity across the range of conversion of the informative parameter $\mathbf{M}_{P}$ are a derivative of the second-order equation of the conversion (1) where $S_{M_{P}}^{\prime}=\partial^{2} N_{M y} / 2 \partial M_{P}^{2}$ in this case is zero.

The coefficients of the influence of the impact value, i.edy. the moment of inertia $J$ on the output parameter of the measurement means of the torque, may be described by analytical expressions such as

$$
\begin{aligned}
& \beta_{0 J}=\frac{\partial N_{\mathrm{My}}}{\partial J}=\frac{A K 1}{J}\left(S+\frac{B\left(M_{P}-M_{\mathrm{CC}}\right)}{J}\right) \\
& \times\left[-\frac{i \eta B}{((J C-D) / \omega)^{2}}-\frac{1}{(J C-\mathrm{D})}\right] \\
& -\frac{A \times B \times K 1 \times\left(M_{P}-M_{\mathrm{CC}}\right)}{(J C-D) J^{2}}, \\
& \beta_{0 J}^{\prime}=\frac{\partial^{2} N_{\mathrm{My}}}{2 \partial J^{2}} \\
& =A K 1\left(S+\left(B\left(M_{P}-M_{\mathrm{CC}}\right) / J\right)\right) \\
& \times \frac{\left[\begin{array}{c}
\left(2 B^{2}(i \eta)^{2}\right) /\left(((J C-D) / \omega)^{3} \omega^{-1}\right) \\
+(4 i \eta B) /\left(((J C-D) / \omega)^{2}\right.
\end{array}\right]}{2 J^{2}} \\
& +\frac{A K 1}{J^{2}}\left[\frac{B^{2} i \eta\left(M_{P}-M_{\mathrm{CC}}\right)}{((J C-D) / \omega)^{2} J}+\frac{\left(S+\left(B\left(M_{P}-M_{\mathrm{CC}}\right) / J\right)\right)}{(J C-D)}\right. \\
& \left.+\frac{2 B\left(M_{P}-M_{\mathrm{CC}}\right)}{(J C-D) J}\right] \text {. }
\end{aligned}
$$

The coefficient of joint influence of the informative parameter and the impact value, that is the moment of inertia $J$ on the nominal sensitivity $S_{\mathbf{M} p}$ of the torque
MM, may be described by an analytical expression with the form

$$
\begin{aligned}
\alpha_{0 J} & =\frac{\partial^{2} N_{\mathrm{My}}}{\partial M_{P} \partial J} \\
& =\frac{A K 1}{J}\left(-\frac{B^{2} i \eta}{((J C-D) / \omega)^{2}}-\frac{2 B}{(J C-D) J}\right) .
\end{aligned}
$$

The analytical expression of the nominal function of the conversion of the torque MM taking into account equation (3) may now be stated in the form

$$
N_{N}=S_{M_{P}} M_{P}=A\left(\frac{K 1 B}{J(J C-D)}+1\right) M_{P} .
$$

The analytical expression which describes the absolute multiplicative error of the torque MM $\Delta N_{\mathrm{MJ}}$ (Tab. 1) under the condition of the change of the moment of inertia $J$ (as an impact value) on the value where $J=J_{N}+\Delta J$ (where $J_{N}$ is the nominal value of moment of inertia), now takes the form

$$
\begin{aligned}
& \Delta N_{\mathrm{MJ}}=\frac{\partial S_{M_{P}}}{\partial J} M_{P} \Delta J=\frac{A B K 1}{J_{N}+\Delta J} \\
& \times\left(-\frac{C}{\left(\left(J_{N}+\Delta J\right) C-D\right)^{2}}-\frac{\left(J_{N}+\Delta J\right)^{-1}}{\left(\left(J_{N}+\Delta J\right) C-D\right)}\right) M_{P} \Delta J .
\end{aligned}
$$

The analytical expression describing the absolute additive error of the torque $\mathrm{MM} \Delta N_{a J}$ (Tab. 1) under the conditions where the change in the moment of inertia $J$ (as an impact value) on the value of $\Delta J$, may now be expressed as

$$
\begin{aligned}
\Delta & N_{a J}=\left.\frac{\partial N_{N}}{\partial J}\right|_{M_{p}=\mathrm{const}} \Delta J=A B K 1 M_{p} \Delta J \\
& \times\left(-\frac{C\left(J_{N}+\Delta J\right)^{-1}}{\left(\left(J_{N}+\Delta J\right) C-D\right)^{2}}-\frac{\left(J_{N}+\Delta J\right)^{-2}}{\left(\left(J_{N}+\Delta J\right) C-D\right)}\right) .
\end{aligned}
$$

The characteristics of change in the obtained metrological models of static characteristics models (1) and (3)-(9), of the torque MM at nominal values for the impact values, are shown in Figures 2-7.

As may be seen in Figure 2, the static characteristics of conversion equation (1) of the torque MM are linear over the entire range of measurement. The sensitivity of the MM (Fig. 3) is constant, and therefore the scale of such a MM is even.

It may be seen in Figure 4 that the absolute multiplicative error of the torque MM under the conditions of deviation of the impact value $J$ will acquire the highest values when measuring the torque at the upper limits of the measuring range. The range of fluctuation in the multiplicative error under the conditions of deviation of the impact value $J$, from its nominal value, is 500 denary pulses at the value of the maximum output code of 5000 pulses, which equals $10 \%$. 


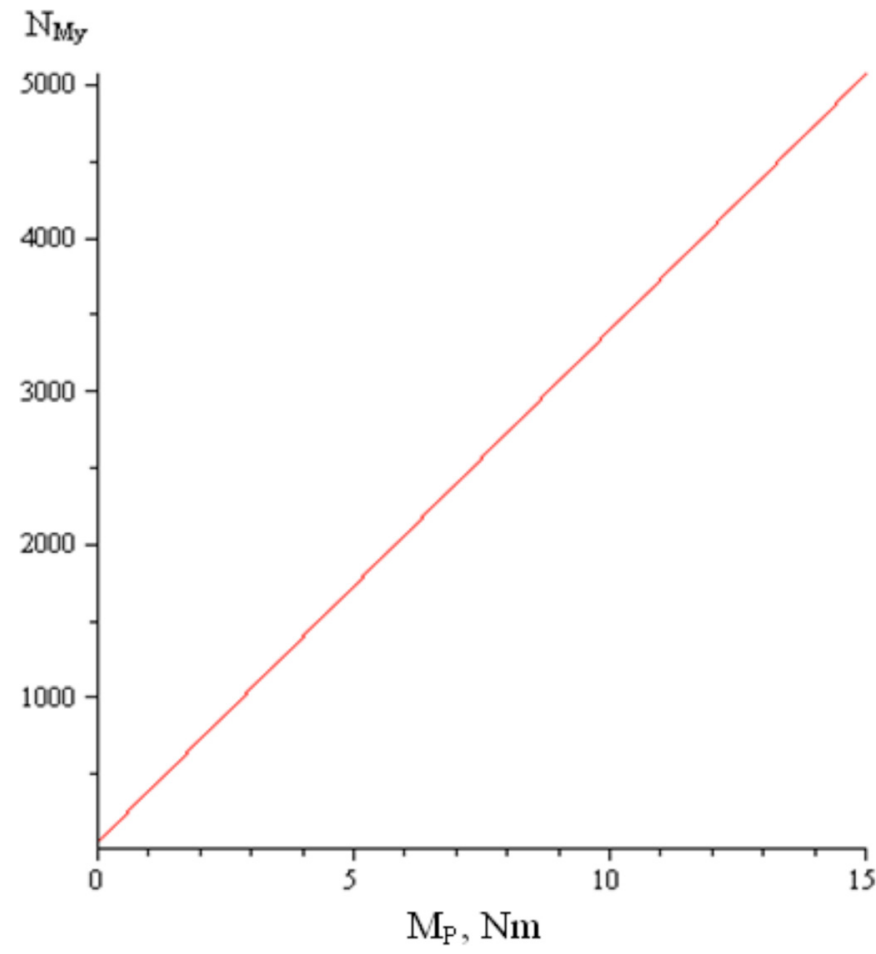

Fig. 2. Static characteristics of the torque MM.

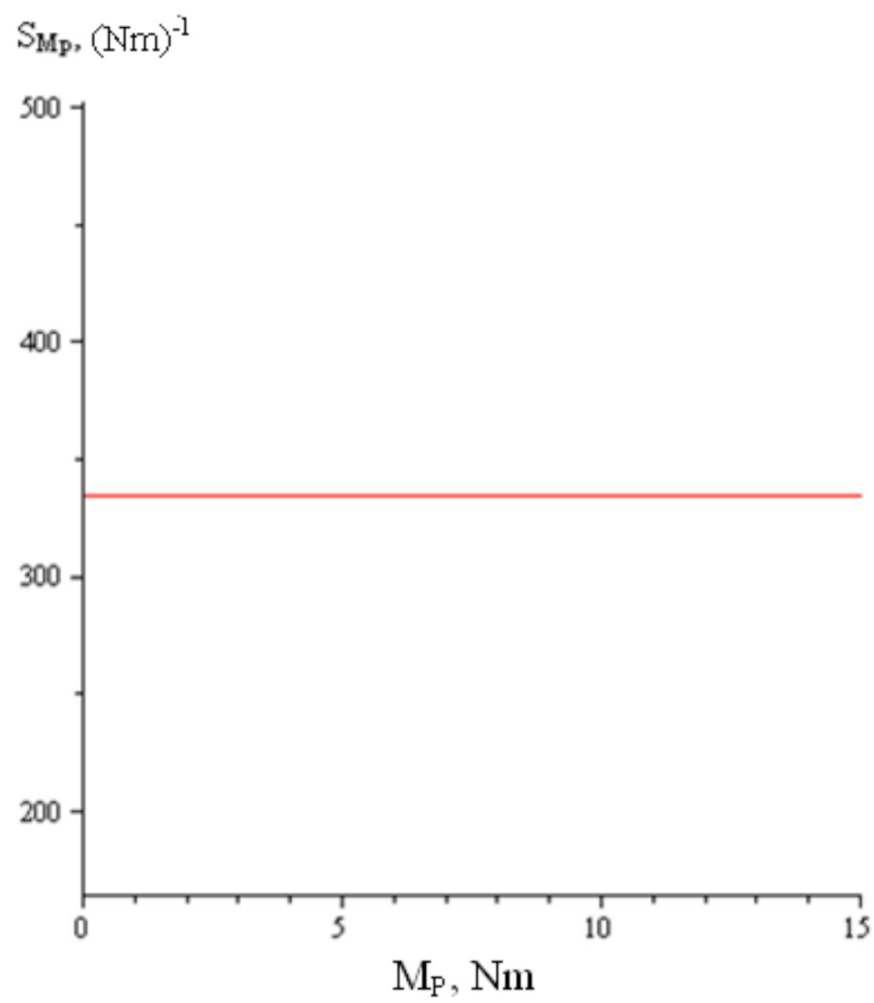

Fig. 3. MM sensitivity.

Therefore, it is feasible that the nominal value of the moment of inertia of the EM when undertaking measurements of the torque of the EM may take into account the above nominal value when calculating the torque in a digital measurement transformer of the torque MM.

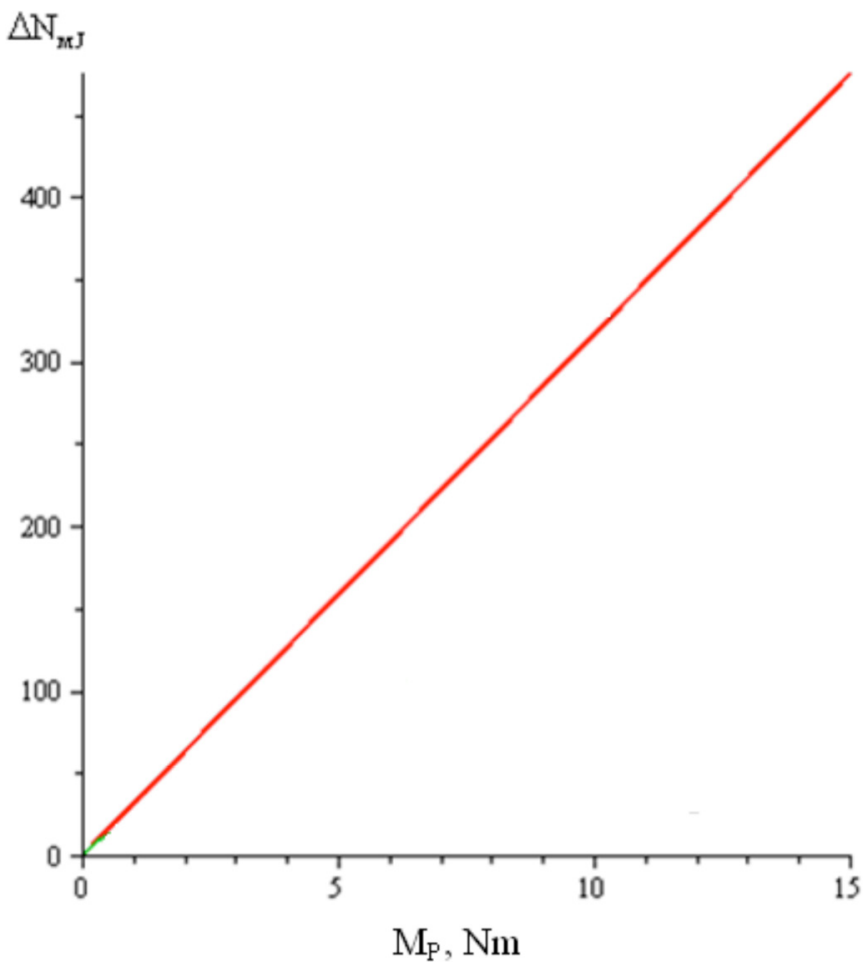

Fig. 4. Description of change in the absolute multiplicative error of the torque MM under the conditions of deviation from the impact value on $\Delta J$.

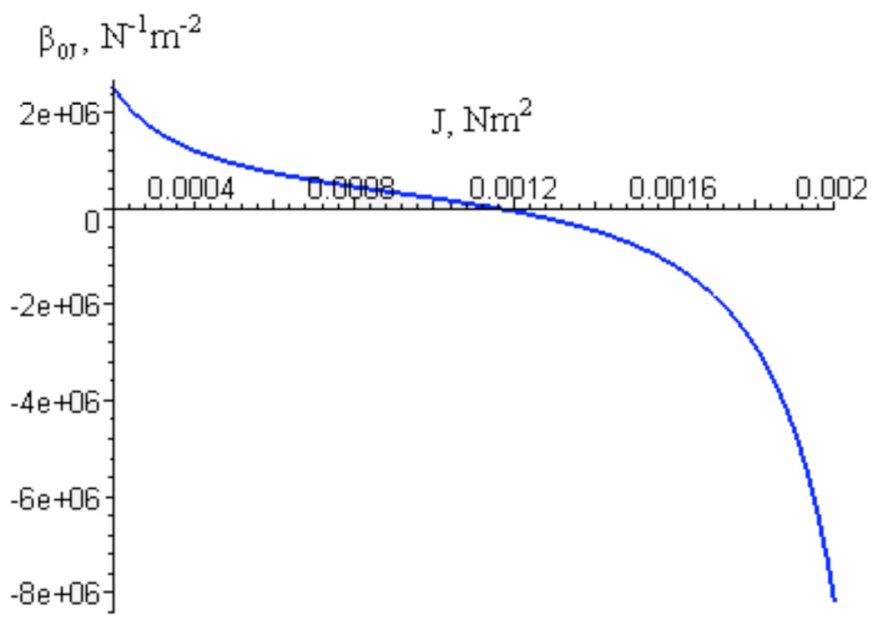

Fig. 5. The coefficient affecting the impact value $J$ on the output value.

In terms of deviation of the impact value $J$ (moment of inertia of the EM) from its nominal value by the amount $\Delta J$, the value of the absolute additive error of the torque $\mathrm{MM}$ increases when the value of the moment of inertia of the EM is more than $0.0014 \mathrm{~N} \mathrm{~m}^{2}$ (Fig. 8).

The discrepancy in the values between the theoretical characteristics (1) and the characteristics of the change in the nominal function of conversion (7) across the entire range of torque measurement differ only by a constant absolute value of the decimal base $\Delta N_{\mathrm{Mp}}$ not exceeding 57 pulses. Taking into account that in the range of the 


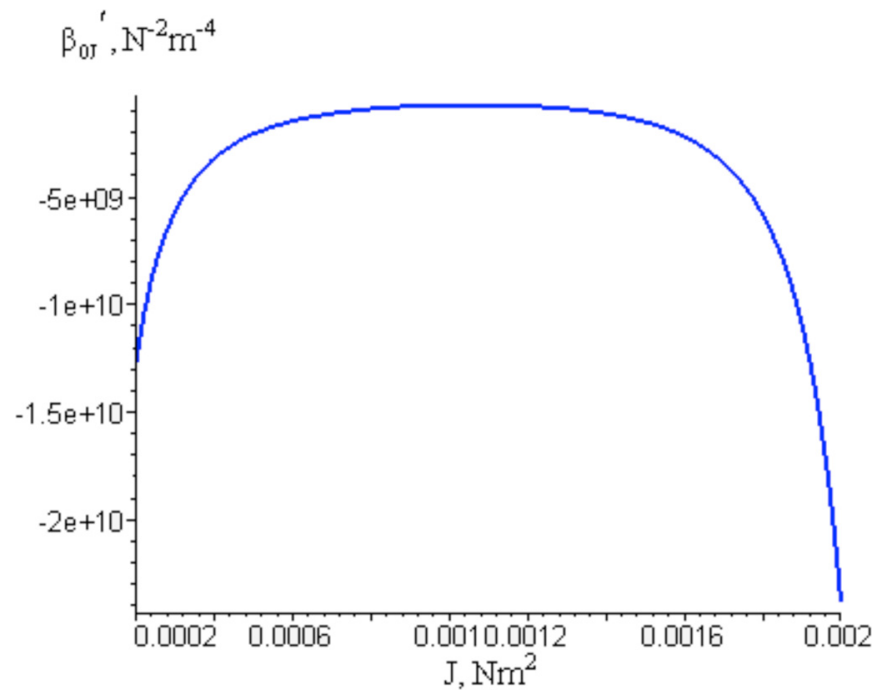

Fig. 6. The speed of change of the impact coefficient of the impact value $J$ on the output value.

conversion of the measurement means from 0 to $15 \mathrm{~N} \mathrm{~m}$, the maximum value of the output value in the decimal code $N_{\mathrm{My}}$ corresponds to 5000 pulses (Fig. 2), the difference of 57 denary pulses will match the decimal value of the torque of $0.17 \mathrm{~N} \mathrm{~m}$. This difference may be taken into account when processing the measurement results by a modification to the specified value of the decimal base.

To represent the obtained accuracy characteristics in accordance with international standards of quality evaluation measurements [4,5], the method of recalculation of the additive and multiplicative errors in the MM in the instrumental component of the uncertainty is set out below.

\section{Evaluation of uncertainty on the basis of additive and multiplicative errors}

When using the above-proposed methods to obtain metrological models for additive and multiplicative errors the issue of how to recalculate the respective components of such errors in accordance with international measurement uncertainty practise of an instrument's uncertainty arises.

We may perform the recalculation of the additive and multiplicative components of the error in the MM in the instrumental component ofuncertainty according to the formula below.

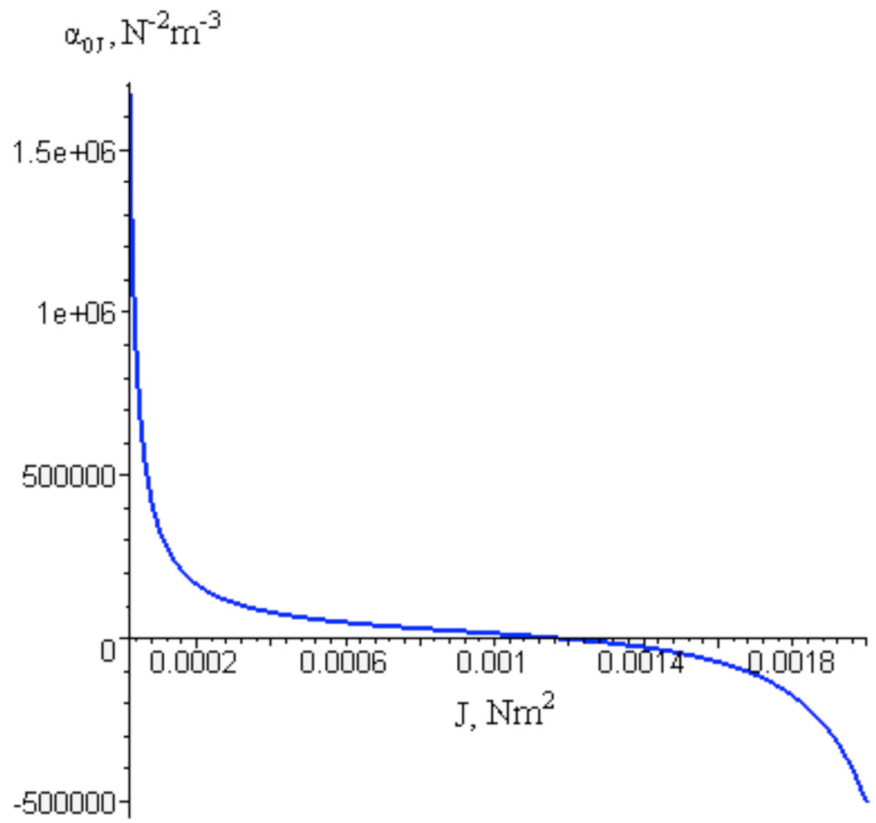

Fig. 7. Characteristics of the change in the coefficient of the cumulative impact of the informative parameter and the impact value $J$ on the value $\Delta J$.

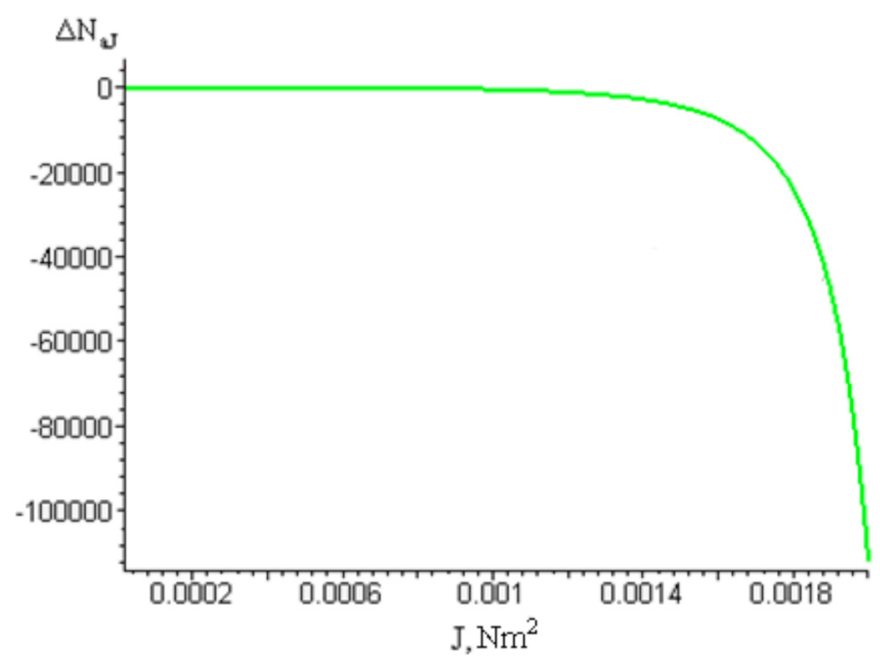

Fig. 8. The boundary of the change in the absolute additive error of the torque MM under the conditions of the deviation of the impact value on $\Delta J$.

$$
\begin{aligned}
u_{\text {Binst }} & =\sqrt{\left(\frac{\partial N}{\partial \eta}\right)^{2} u^{2}(\Delta \eta)+\left(\frac{\partial^{2} N}{2 \partial \eta^{2}}\right)^{2}(2 \Delta \eta u(\Delta \eta))^{2}+\left(\frac{\partial^{2} N}{\partial x \partial \eta}\right)^{2} u^{2}(\Delta x) u^{2}(\Delta \eta)} \\
& =\sqrt{\left[\left(\frac{\partial N}{\partial \eta}\right)^{2}+\left(\frac{\partial^{2} N}{\partial \eta^{2}} \Delta \eta\right)^{2}\right] u^{2}(\Delta \eta)+\left(\frac{\partial^{2} N}{\partial x \partial \eta}\right)^{2} u^{2}(\Delta x) u^{2}(\Delta \eta)} \\
& =\sqrt{\left[\left(\frac{\partial N}{\partial \eta}\right)^{2}+\left(\frac{\partial^{2} N}{\partial \eta^{2}} \Delta \eta\right)^{2}\right] \frac{\Delta \eta^{2}}{12}+\left(\frac{\partial^{2} N}{12 \partial x \partial \eta} \Delta x \Delta \eta\right)^{2}} .
\end{aligned}
$$


This issue may be addressed by applying the model equation of the conversion of the MM to the Taylor series, from which we may obtain the components of the additive and multiplicative errors which may be described by the expressions:

$$
\begin{gathered}
\Delta N_{a}=\frac{\partial N}{\partial \eta}\left(\eta-\eta_{0}\right)+\frac{\partial^{2} N}{2 \partial \eta^{2}}\left(\eta-\eta_{0}\right)^{2}=\frac{\partial N}{\partial \eta} \Delta \eta+\frac{\partial^{2} N}{2 \partial \eta^{2}} \Delta \eta^{2} \\
\Delta N_{m}=\frac{\partial^{2} N}{\partial x \partial \eta}\left(x-x_{0}\right)\left(\eta-\eta_{0}\right)=\frac{\partial^{2} N}{\partial x \partial \eta} \Delta x \Delta \eta
\end{gathered}
$$

where $N$ is the output value of the MM; $x$ is the measured input value; $x_{0}$ is the nominal value of the input value (the value that under normal conditions meets the requirements for calibration of the MM); $\eta$ is the impact value; and $\eta_{0}$ is the nominal value of the impact value.

In order to recalculate the additive and multiplicative components of the error of the MM in the instrumental component of uncertainty necessary to determine the uncertainty of the measured input value $u(\Delta x)$ and the impact value $u(\Delta \eta)$ for the upper and lower boundaries $\left(\left[x_{-} ; x_{+}\right] i\left[\eta_{-} ; \eta_{+}\right]\right)$, having assumed a possible interpretation of the law, we may assess the distribution of error within the limits.

If nothing is known about the interpretation of the law of the distribution of the error within the limits, in accordance with international recommendations on the evaluation of measurement uncertainty, we should assume that there is equal probability that such an error may have any value with the accepted range, and hence we may adopt rectangular probability density function distribution. If the form of the distribution law of error within the boundaries of the studied values is known, then the uncertainty may be determined by taking into account the form of the distribution law based on the known formulae presented in $[5,15,16]$.

We may now consider the case where information about the nature of the distribution is absent $[15,16]$, and in this case we should initially determine the uncertainty of the input and output values in accordance with the formulae:

$$
u(\Delta x)=\frac{\Delta x}{\sqrt{12}} ; u(\Delta \eta)=\frac{\Delta \eta}{\sqrt{12}} .
$$

Let us now introduce the following factors for the influence $\vec{\eta}$ of the impact values on the output parameter value $N$ :

$$
\begin{gathered}
\sum_{i=1}^{n} \frac{\partial N}{\partial \eta_{i}}=\sum_{i=1}^{n} \beta_{0 i}, \\
\sum_{i=1}^{n} \frac{\partial^{2} N}{2 \partial \eta^{2}{ }_{i}}=\sum_{i=1}^{n} \beta_{0 i}^{\prime},
\end{gathered}
$$

and the coefficient of the influence of the impact values on the nominal sensitivity of the MM

$$
\sum_{i=1}^{n} \frac{\partial^{2} N}{\partial x \partial \eta_{i}}=\sum_{i=1}^{n} \alpha_{0 i}
$$

The model equation that will determine the instrumental component of the variance at the impact values (more than one impact value) takes the form

$$
\begin{aligned}
u_{\text {Binst }}^{2} & =\sum_{i=1}^{n} \beta_{0 i}^{2} u^{2}\left(\Delta \eta_{i}\right)+4 \sum_{i=1}^{n}{\beta^{\prime}}_{0 i}^{2} \Delta \eta_{i}^{2} u^{2}\left(\Delta \eta_{i}\right) \\
& +\sum_{i=1}^{n} \alpha_{0 i}^{2} u^{2}(\Delta x) u^{2}\left(\Delta \eta_{i}\right) .
\end{aligned}
$$

When we consider equation (12), the analytical expression (17) for $n$ impact values will take the form

$$
\begin{aligned}
u_{\text {Binst }}^{2} & =\frac{\sum_{i=1}^{n}\left(\beta_{0 i} \Delta \eta_{i}\right)^{2}}{12}+\frac{\sum_{i=1}^{n}\left(\beta^{\prime}{ }_{0 i} \Delta \eta_{i}^{2}\right)^{2}}{3} \\
+ & \frac{\sum_{i=1}^{n}\left(\alpha_{0 i} \Delta x \Delta \eta_{i}\right)^{2}}{12^{2}}
\end{aligned}
$$

For a single impact value the expression (18) takes the form

$$
u_{\text {Binst }}^{2}=\frac{\left(\beta_{0} \Delta \eta\right)^{2}}{12}+\frac{\left(\beta_{0}^{\prime} \Delta \eta^{2}\right)^{2}}{3}+\left(\frac{\alpha_{0} \Delta x \Delta \eta}{12}\right)^{2} .
$$

Thus, the method of estimating the standard uncertainty of type B based on the additive and multiplicative components of the error is reduced to calculation of the dispersion according to formulas (13) or (17).

This proposed method for the recalculation of the component of the instrumental error of the torque MM ((8) and (9)) in the instrumental component of uncertainty may now tested with practical calculations.

In as much as it has been established that the difference between the theoretical equation of the conversion (1) of the torque MM and the obtained nominal function of the conversion (7) across the whole measurement range of the torque is $0.17 \mathrm{~N} \mathrm{~m}$. Then the standard uncertainty of type B, that is introduced by the differences between the nominal function of the conversion and the model equation of the measurement may be determined by the expression (12) thus $u(\Delta M)=0.17$ / $\sqrt{12}=0.05 \mathrm{Nm}$.

Uncertainty of type $\mathrm{B}$, that is introduced by the deviation by the moment of inertia of the rotor of the EM $\Delta J=J-J_{N}=10^{-5} \mathrm{Nm}^{2}$, as an impact value under the assumption of evenness in the distribution law, is $u(\Delta J)=10^{-5} / \sqrt{12}=2.89 \times 10^{-6} \mathrm{~N} \mathrm{~m}^{2}$. In order to recalculate the additive and multiplicative components obtained for the error in the torque MM in the instrumental component of $t$ uncertainty in the measurement, let us introduce the obtained values of the uncertainties $u(\Delta \mathbf{M})$ and $u(\Delta J)$, and maximum values of influence factors (4)-(6) at nominal parameters of the impact values shown in Figures 5-7 to the analytical expression (17). The result is the value of the instrumental component of the variance 
in the torque MM, shown by the expression

$$
\begin{aligned}
& u_{\text {Binst }}^{2}(\Delta M, \Delta J) \\
& \quad=\beta_{0 J}^{2} u^{2}(\Delta J)+4{\beta^{\prime}}_{0 J}^{2} \Delta J^{2} u^{2}(\Delta J) \\
& \quad+\alpha_{0 J}^{2} u^{2}(\Delta M) u^{2}(\Delta J) \\
& \quad=\left(-8 \times 10^{6} \mathrm{~N}^{-1} \mathrm{~m}^{-2}\right)^{2}\left(2.89 \times 10^{-6} \mathrm{~N} \mathrm{~m}^{2}\right)^{2} \\
& \quad+4\left(-2.5 \times 10^{10} \mathrm{~N}^{-2} \mathrm{~m}^{-4}\right)^{2}\left(0.5 \mathrm{~N} \mathrm{~m}^{2}\right)^{2}\left(2.89 \times 10^{-6} \mathrm{~N} \mathrm{~m}^{2}\right)^{2} \\
& \quad+\left(1.65 \times 10^{6} \mathrm{~N}^{-2} \mathrm{~m}^{-3}\right)^{2}(0.05 \mathrm{~N} \mathrm{~m})^{2}\left(2.89 \times 10^{-6} \mathrm{~N} \mathrm{~m}^{2}\right)^{2} \\
& \quad=536.68
\end{aligned}
$$

Taking the square root of the calculated value of the variance (20), we obtain an instrumental component of uncertainty that is 23.17 of the denary pulses of the output signal of the torque MM within therange of measurement from 0 to $15 \mathrm{Nm}$ at a maximum value of decimal pulses at the upper boundary of measurements at 5000 (Fig. 2). Having recalculated the obtained value of uncertainty for the denary pulses into an absolute value of the measured value $(15 \mathrm{~N} \mathrm{~m}$ $\times 23.17 / 5000=0.07 \mathrm{~N} \mathrm{~m})$, we obtain the value of standard uncertainty of type B (Tab. 1), which is $0.07 \mathrm{~N} \mathrm{~m}$ under the conditions where the deviation of the moment of inertia of the rotor of the EM from its nominal value by $10^{-5} \mathrm{~N} \mathrm{~m}^{2}$.

Thus, as a result of our study, we have shown that the standard uncertainty of type $B$ of the torque $M M$ is $0.07 \mathrm{Nm}$ within a measurement from 0 to $15 \mathrm{Nm}$ with an acceptable deviation of the impact value (moment of inertia) of its nominal value by $10^{-5} \mathrm{~N} \mathrm{~m}^{2}$.

\section{Conclusions}

To represent standard uncertainty of type B, which is caused by the presence of additive and multiplicative errors in the MM, mathematical expressions are proposed that allow recalculation of these errors to standard uncertainty. The mathematical expressions were tested using the example of the recalculation of additive and multiplicative errors of the MM of the torque in the standard uncertainty of type B. These analytical expressions of additive and multiplicative errors were derived from the decomposition of the transformation equation of the MM of the torque into a Taylor's Series. The characteristics of the change in the additive and multiplicative errors are presented in Figures 4 and 8 , respectively. As a result of the recalculation of these type B instrument uncertainties using formula (20) we determined that in the measurement range from 0 to $15 \mathrm{Nm}$ that the standard uncertainty was $0.07 \mathrm{Nm}$. The proposed technique of the recalculation of the additive and multiplicative errors in the standard uncertainty of type B allows the research results to represent the characteristics of the precision of measuring means in accordance with international standards for evaluating the uniformity of measurements.
The mathematical expressions (10)-(19) used for the recalculation of additive and multiplicative errors into the standard uncertainty of type B can be used not only for the uniform distribution of the limits of impact values, but also for other distribution laws.

Thus, the resulting mathematical tools and characteristics of change of the basic static metrological MM for torque give us the opportunity to identify the causes of increase in error in the measurement of torque in EMs. This enables us to explore how to diminish or eliminate them, and also to synthesise torque MM with standardised metrological characteristics.

\section{References}

1. ISO 9000:2005, Quality management systems - Fundamentals and vocabulary (ISO, Switzerland, 2005)

2. ISO 9001 2008, Quality management systems - Requirements (ISO, Switzerland, 2008)

3. ISO/TR 10017:2003, Guidance on statistical techniques for ISO 9001:2000 (ISO, Switzerland, 2003)

4. ISO 5725-1:1994/Cor 1:1998, Accuracy (trueness and precision) of measurement methods and results - Part 1: General principles and definitions (ISO, Switzerland, 1994/1998)

5. ISO/IEC 17025:2005, General requirements for the competence of testing and calibration laboratories (ISO, Switzerland, 2005)

6. S. Eichstädt, C. Elster, Reliable uncertainty evaluation for ODE parameter estimation - a comparison, J. Phys.: Conf. Ser. 490, 012230 (2014)

7. O.M. Vasilevskyi, Advanced mathematical model of measuring the starting torque motors, Tekhn. Elektrodin. 6, 76 (2013)

8. V. Subrahmanyan, Electric drives (McGraw-Hill, USA, 1996), p. 715

9. L. Klaus, T. Bruns, M. Kobusch, Modelling of a dynamic torque calibration device and determination of model parameters, ACTA IMEKO 3, 14 (2014)

10. O.M. Vasilevskyi, A frequency method for dynamic uncertainty evaluation of measurement during modes of dynamic operation, Int. J. Metrol. Qual. Eng. 6, 202 (2015)

11. D. Röske et al., Metrological characterization of a $1 \mathrm{~N} \mathrm{~m}$ torque standard machine at PTB, Metrologia 51, 87 (2014)

12. F. Tegtmeier et al., Investigation of transfer standards in the highest range up to $50 \mathrm{MN}$ within EMRP project Sib 63, in XXI IMEKO World Congress, Prague, Czech Republic, 2015(2015)

13. C. Schlegel, H. Kahmann, R. Kumme, MN·m torque calibration for nacelle test benches using transfer standards, ACTA IMEKO 5, 12 (2016)

14. O.M. Vasilevskyi, Calibration method to assess the accuracy of measurement devices using the theory of uncertainty, Int. J. Metrol. Qual. Eng. 5, 403 (2014)

15. GUM, Guide to the expression of uncertainty in measurement (ISO, Genf, 2008)

16. O.M. Vasilevskyi, Methods of determining the recalibration interval measurement tools based on the concept of uncertainty, Tekhn. Elektrodin. 6, 81 (2014) 